\title{
Microchannel Thermocured Silicone Rubber
}

\author{
Nataliya Zolotareva $\cdot$ Vladimir Semenov
}

Received: 29 April 2014 / Accepted: 27 August 2014 / Published online: 19 October 2014

(C) Springer Science+Business Media Dordrecht 2014

\begin{abstract}
Silicone rubber penetrated by a network of microchannels with diameters $10-600 \mu \mathrm{m}$ and lengths $2-30 \mathrm{~mm}$ was formed by washing away of whiskers of paminobenzoic acid from a thermocured polymethylphenylsiloxane composition.
\end{abstract}

Keywords Silicone rubbers · Whiskers · Scaffolds

\section{Introduction}

Porous polymers $[1,2]$ are used as membranes and implants in medicine [2,3] and technology. The silicones take a special position. They form a considerable quantity of materials with such unique properties as transparency, biocompatibility, high elasticity and thermal stability, low glass transition temperature and high gas permeability. There are several methods of forming porous structures. One of them consists of obtaining a polymer filled with microparticles of water-soluble inorganic salts. Washing with water leads to the formation of pores. Creation of microchannels is carried out photolithography [4], capillary force lithography [5], mould and hot embossing [6,7], sputtering on a sacrificial polymer fiber [8] and a hot roller embossing process [9]. These ways provide products with a strictly set network of microchannels. Research in this area was initiated by the introduction of microfluidic technology in chemistry,

N. Zolotareva $(\bowtie) \cdot$ V. Semenov

G.A. Razuvaev Institute of Organometallic

Chemistry, Russian Academy of Sciences, 603950 Nizhnii Novgorod, Russian Federation

e-mail: natkamel@yandex.ru medicine and biology [10]. The studies fall into areas of traditional organic polymers and the organic-inorganic hybrid materials obtainable by a method of sol-gel chemistry.

Silicones [11] are widely used in medicine as soft implants. The presence of pores and channels will provide intergrowth of the implanted material by blood-vessels and tissue. Such materials are called "scaffolds". There is a limited quantity of data [7, 12-14] about the production of microchannel polymers of this class. In the present work we offer a new method of preparation of microchannel polyorganosiloxane (MP) using whiskers as the porogen agent.

Whiskers [15] are a special form of crystal compounds. Natural and artificially obtained whiskers have attracted attention by their high hardness approaching the theoretically possible. They have a great future for high-strength materials [15]. However, these remarkable qualities belong to whiskers of metals, their oxides and their nitrides. Watersoluble whiskers (for example, sodium chloride) are an extremely brittle material and are easily broken at slight stress. In this connection their use as polymer filler is inconvenient or even impossible. Whiskers of some organic compounds can possess high hardness. Especially this relates to compounds with intermolecular hydrogen bonding.

\section{Experimental}

\subsection{Materials}

p-Aminobenzoic acid (purity $>99 \%$ ) was purchased from Sigma-Aldrich and used without further purification. We used a liquid silicone rubber as a polymeric matrix. The thermocured silicone composition consisted of vinyl (《silicone $\mathrm{A} \gg)$ and hydride (《silicone $\mathrm{B} \gg)$ 
components [16, 17]. Silicone A is a mixture of poly(dimethyl)(methylphenyl)siloxane with terminal trivinylsiloxy groups and oligomethylvinylsiloxane at a weight ratio of 100:5. Silicone B is a oligomethylhydrosiloxane. The catalyst is a $1 \%$ solution of the platinum complex in oligovinylsiloxane, which can be obtained by interaction of $\mathrm{H}_{2} \mathrm{PtCl}_{6} \cdot 6 \mathrm{H}_{2} \mathrm{O}$ with tetravinyldimethylsiloxane. The ratio of silicone A and silicone $\mathrm{B}$ is 10:1. The catalyst is $1 \%$ of the total mass. The viscosity of a thermocured silicone composition after mixing of components A and $\mathrm{B}$ is 6000 - $8000 \mathrm{St}$, refractive index $\mathrm{n}_{\mathrm{d}}^{20} 1.50$.

\subsection{Preparation of Whiskers}

We used p-aminobenzoic acid (ABA) to obtain the whiskers. Crystals were grown from the hot saturated aqueous solutions of ABA. The rapid cooling of the bulb $(1 \mathrm{~h})$ led to the formation of many thin whiskers. Slow cooling of the flask $(3-4 \mathrm{~h})$ promoted the lengthening and thickening of the crystals. The largest crystals were obtained in the Dewar vessel with hot water (during $10-12 \mathrm{~h}$ ). Thus, we could produce four types of ABA of different sizes depending on the crystallization conditions: with lateral dimensions $10-20,50-70,200-300,500-600 \mu \mathrm{m}$ and lengths $2-3,5-10,10-20,20-30 \mathrm{~mm}$, respectively. The aqueous suspension of crystals was filtered and dried in a dry box at a temperature $70-80^{\circ} \mathrm{C}$.

\subsection{Preparation of MP}

Microchannel silicone with a chaotic arrangement of channels was obtained in a teflon vessel. The whisker-filled teflon vessel was coated with a liquid silicone composition and stored for $10-12 \mathrm{~h}$. The whiskers comprised 4-5\% of the total mass of the samples. The liquid composition filled with large crystals was completely deaerated during this time. The silicone matrix after curing $\left(100{ }^{\circ} \mathrm{C}, 1 \mathrm{~h}\right)$ is transparent and does not contain air bubbles. However air is not removed completely from the composition with small crystals. Deaeration of the liquid composition filled with small crystals was carried out by vacuum.

Whiskers were washed away from the polymer matrix by ethanol. The application of distilled water for this purpose showed unsatisfactory results because of the well-known hydrophobic properties of polyorganosiloxanes. Ethanol moistens well both the acid crystals and the polymeric matrix. Consequently the polymeric matrix slightly swells in boiling alcohol and becomes turbid. The largest threads can be washed by long storage (10 days) of the sample in ethanol at ambient temperature. However residual fine threads are washed slowly. The rate of washing is retarded by increasing the thickness of the sample. The most effective removal of crystals from a matrix is carried out by long
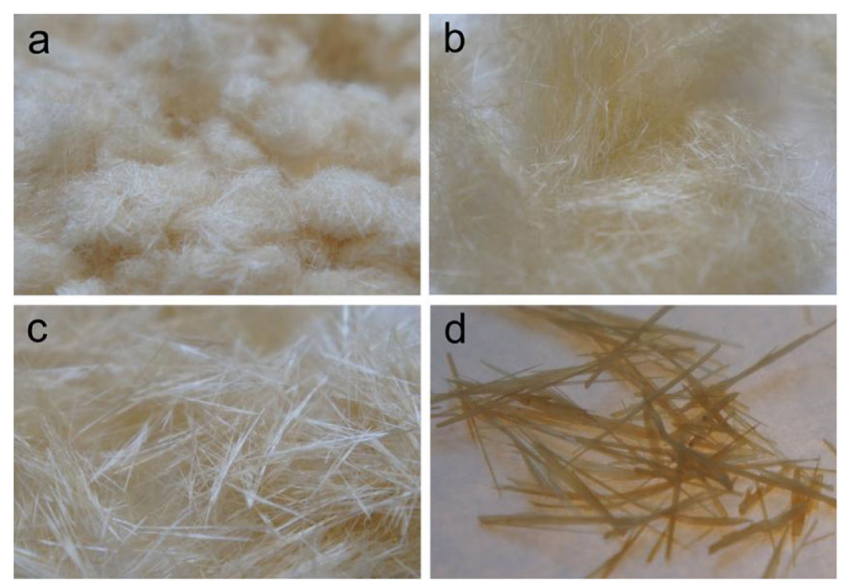

Fig. 1 The appearance of 4 types of ABA after recrystallization (lateral dimension, $\mu \mathrm{m} /$ length, mm: a - 10-20 / 2-3, b - 50-70/5-10, c 200-300/10-20, d - 500-600/20-30)

ethanol extraction $(10-15 \mathrm{~h})$ in a Soxhlet's apparatus. The swollen samples are turbid; however after drying in free air they become transparent.

\subsection{Instrumentation}

$\mathrm{X}$-ray diffraction patterns for the samples were measured using an X-ray diffractometer «Shimadzu XRD-7000». The shapes and sizes of whiskers and structure of MP were imaged using an optical microscope "Leica DM 4000M". The specific surface area of MP was measured by the BETmethod using a gas chromatograph "Tzwet-500". The determination of microimpurities in MP was carried out using a chromato-mass-spectrometer "Polaris Q/Trace GC Ultra". The surface of ABA was investigated with an atomic force microscope "Solver PRO-M" (NT-MDT, Russia). For analyses of MP a differential scanning calorimeter "DSC204F1 Phoenix" (Netzsch Geratebau, Germany) was used.

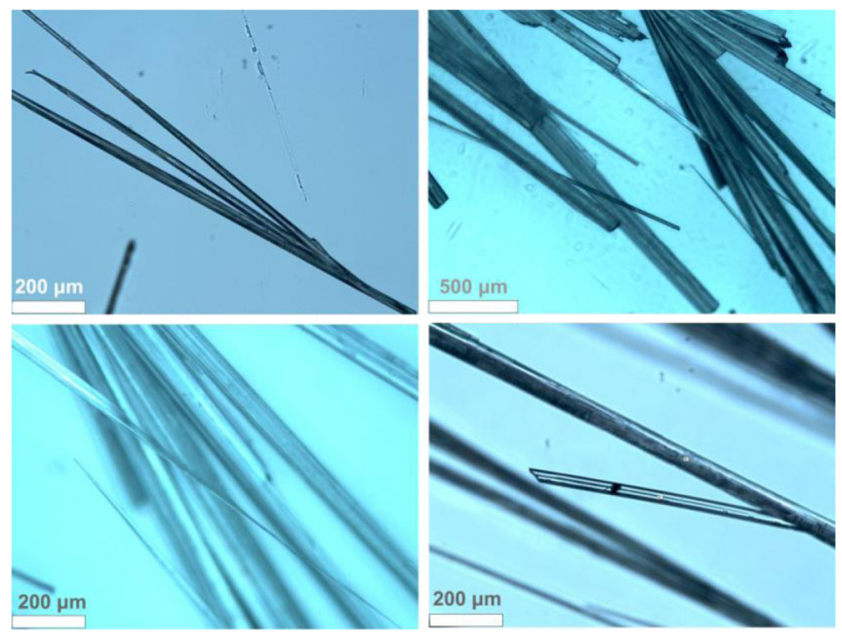

Fig. 2 Microscopic images of ABA whiskers 


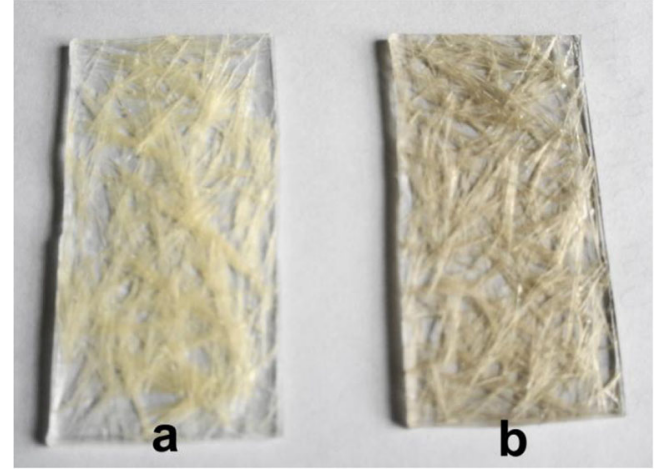

Fig. 3 The microchannel silicone rubber: a - with ABA crystals (type $c$, Fig. 1), b - free from ABA crystals

\section{Results and Discussion}

In Fig. 1 four types of obtained ABA grown in water are demonstrated. The crystals of ABA are strong enough and can be easily manipulated. Moreover A have a sufficiently large relative density $(1.367-1.395)$ [18-21] and therefore crystals do not float on the surface of the liquid silicone composition during the process of mixing. Very polar ABA crystals are well wetted with the uncured silicone rubber. As shown by optical microscopy derived whiskers are individual or compactly accreted thin threads of right-angled section (Fig. 2). For samples of large and average sizes $(b-d$, Fig. 1) we observed splitting on terminal parts.

$\mathrm{X}$-ray data shows that derived samples are both individual whiskers and crystalline aggregates. The presence of spots on the X-ray diffraction patterns shows that tested samples are monocrystals. Splitting of spots on other Laue patterns testifies to the block structure of the whiskers (see Supporting information, 1). According to AFM the surface of the whiskers is rather smooth (see Supporting information, 2). ABA is used as an antiviral drug, interferon inductor, anesthetic and antioxidant. It does not have embriotoxity, teratogenic and mutagenic activity.

Figure 3 shows the appearance of MP samples with a thickness of $3 \mathrm{~mm}$. Figure 4 demonstrates optical images of MP without ABA crystals. Note that the channels represent the complete imprint of the whiskers (Fig. 5). To obtain the optical image (Fig. 5a) a sample of MP was cut along the
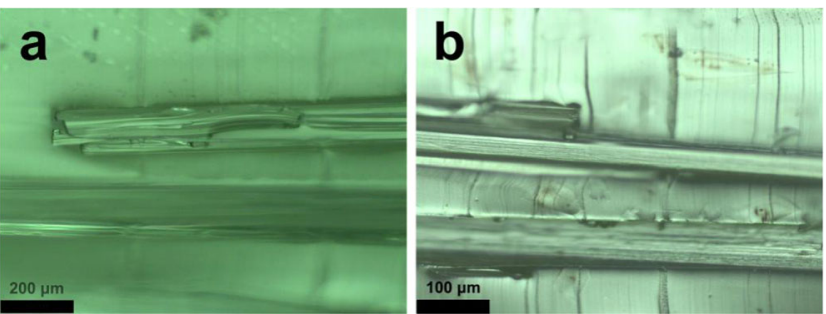

Fig. 5 Microscopic images of longitudinal section of MP (a - pure MP, b - Co-coated silicon)

canals. To increase the quality of the images the slice of MP was coated with dicobalt octacarbonyl vapor in vacuum at a temperature of $75^{\circ} \mathrm{C}$ (Fig. 5b).

The application of toluene as an extractive solvent has shown that it can be used as an immersion liquid. The refractive indexes of toluene and phenyl containing polysiloxane practically are equal. Toluene causes the slow swelling of a matrix and filling of the microchannels. The network of capillaries is clearly visible after $15-20$ min of the sample dipping in solvent (Fig. 6).

Full filling of the channels takes place after $25-30 \mathrm{~h}$, the sample bulks up and expands approximately by 1.5 times. The observable effect was used for measurement of the total volume $(\mathbf{V})$ of microchannels. Value $\mathrm{V}$ is found to be 0.69 $\mathrm{cm}^{3} / \mathrm{g}$ for the sample filled with crystals of cross sectional dimensions $10-20 \mu \mathrm{m}$. The specific surface area measured by the BET-method is $4.6 \mathrm{~m}^{2} / \mathrm{g}$.

The degree of openness of the channels has been defined by coloring of samples in Rodamine-6G dissolved in ethanol. Filling of the cavities was carried out in vacuum. The solution of Rodamine-6G painted only channels (see Supporting information, 3). Penetration into the intermolecular space of the silicone matrix was not observed. The tests have shown that channels were open and they were quickly filled with a dye solution. Attempts to use for these purposes toluene solutions of silicon phthalocyanines $\mathrm{Me}_{3} \mathrm{SiO}-$ [PcSi]-OSiMe ${ }_{3}$ and $\mathrm{Me}_{3} \mathrm{SiO}-[\mathrm{PcSi}]-\mathrm{O}-[\mathrm{PcSi}]-\mathrm{OSiMe}_{3}$ have not been a success [22]. The matrix of the silicone rubber was quickly colored a brilliant blue color at all depths.

The application of polymers in medicine requires a high degree of their purification from residual monomer and
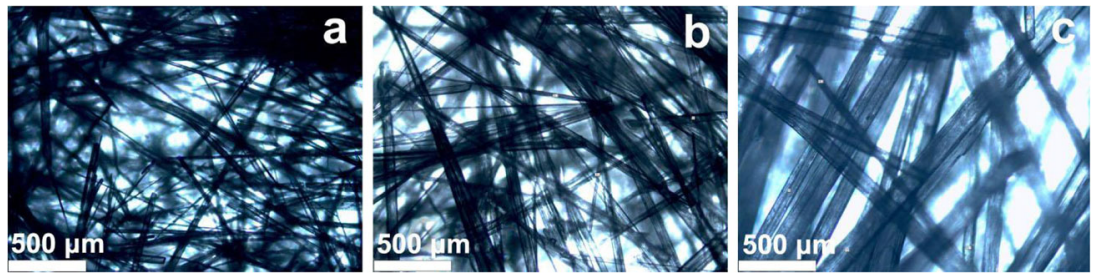

Fig. 4 Microscopic images of MP after the washing away of ABA. The sizes of used whiskers: (lateral dimension, $\mu \mathrm{m} / \mathrm{length}, \mathrm{mm}$ ): a - 10-20 / 2-3, b - 50-70 / 5-10, c - 200-300/ 10-20). It corresponds with the types $a-c$ in Fig. 1 


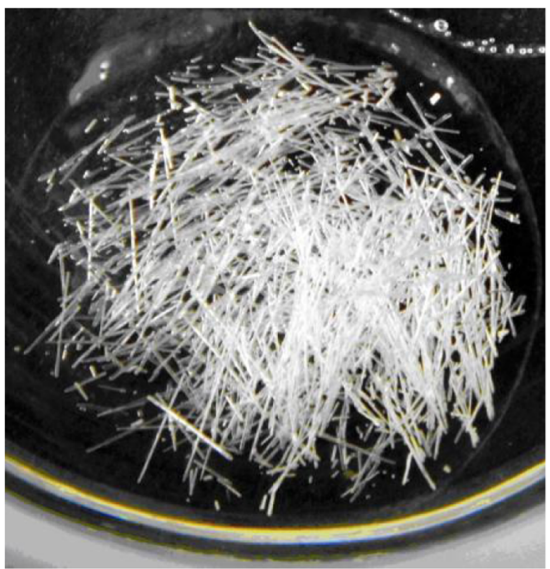

Fig. 6 The network of microchannels of the MP sample in toluene

other microimpurities. Biocompatibility demands a minimum quantity of the defects which occur in a polymer owing to shrinkage during polymerization [23]. Because the silicones are nonshrinking materials, they are characterized by a small content of defects and biocompatibility. However, ABA can play a role of admixture in the case of incomplete washing away of the sample by ethanol. Besides, ABA can contain a small amount of impurities in spite of the double recrystallization. The presence of residual acid and other possible volatile products was defined by heating of microchannel silicon in vacuum at $250{ }^{\circ} \mathrm{C}$ for 1 hour in a glass ampoule with the appendix cooled by liquid nitrogen. Two samples of MP have been tested. The first was washed away with ethanol during $2 \mathrm{~h}$, the second $-15 \mathrm{~h}$. Weight losses were $1.6 \%$ and less than $0.01 \%$, respectively. According to chromatography-mass spectrometry of the ampoule, ABA was as expected. Thus, lasting washing fully frees MP from ABA.

Also, incomplete removal of ABA can cause a change in the physico-chemical characteristics of thermocured silicone. In this regard, samples of MP and pure silicone were studied by the method of differential scanning calorimetry in the range -100 to $+400^{\circ} \mathrm{C}$. DSC curves behave similarly in a wide range of temperatures (see Supporting information, 4). The glass transition is in the range from -60 to $-70^{\circ} \mathrm{C}$. Initial temperature of decomposition for the MP is $350{ }^{\circ} \mathrm{C}$, for pure silicone is $340{ }^{\circ} \mathrm{C}$. Thus, ABA does not affect the structure of the silicone.

\section{Conclusions}

In this manuscript, we report a simple method of formation of microchannels in thermocured silicone rubber. The proposed method consists of the production of polymer filled with whiskers. The washing away of whiskers from silicone rubber leads to the formation of microchannels. We used crystals with lengths 2-30 mm and thicknesses 10-600 $\mu \mathrm{m}$ grown from water solutions of $\mathrm{p}$-aminobenzoic acid. It is known that polysiloxanes are widely used in surgery as flexible stents. The microchannel silicone rubber holds promise for usage as implants.

Acknowledgments This work was supported by the Presidium of the RAS (program "Directed Synthesis of Substances with Specified Properties and Design of Related Functional Materials"). The work was performed using the instrumental base of the Collective Usage Center of Nizhny Novgorod State University "Wave and Quantum Technologies" and Analytical Center of the Institute of Organometallic Chemistry of Russian Academy of Sciences.

\section{References}

1. Wu D, Xu F, Sun B, Fu R, He H, Matyjaszewski K (2012) Design and preparation of porous polymers. Chem Rev 112(7):3959-4015

2. Zhang H, Shen PK (2012) Recent development of polymer electrolyte membranes for fuel cells. Chem Rev 112(5):2780-2832

3. Korzhikov VA, Vlakh EG, Tennikova TB (2012) Polymers in orthopedic surgery and tissue engineering: from engineering materials to smart biofunctionalization of a surface. Polymer Sci A 54(8):585-601

4. Narasimkan J, Papautsky I (2004) Polymer embossing tools for rapid prototyping of plastic microfluidic devices. J Micromech Microeng 14:96-103

5. Lee SH, Kang DH, Kim HN, Suh KY (2012) A bond less fabrication method for homogeneous polymer microchannel by capillary force lithography. 4 Intern. Conf. on Miniaturized Systems for Chemistry and Life Sciences, Groningen, The Netherlands, Book of Abstracts

6. Mikkopadkyay S, Roy SS, Matkur A, Tweedi M, McLaughlin JA (2010) Experimental study on capillary flow through polymer microchannel bends for microfluidic applications. J Micromech Microeng 20(5):055018. (6pp)

7. Gaitan M, Locascio LE (2004) Embedded microheating elements in polymeric microchannel for temperature control and fluid flow sensing. J Res Nat Inst Stand Technol 109(3):335-344

8. Wu X, Reed HA, Wang Y, Rhodes LF, Elce E, Ravikiran R, Shich RA, Henderson CL, Allen SAB, Kohe PA (2003) Fabrication of microchannel using polynorbornene photosensitive sacrificial materials. J Electrochem Soc 150(9):H205-H213

9. Tsao CW, Chen TY, Woon WY, Lo CJ (2012) Rapid polymer microchannel fabrication by hot roller embossing process. Microsyst Technol 18:713-722

10. The micropatterning equipment for chemical technology (2011) Special issue of Rus Khim Zhurnal (Rus Chem J) LV(2)

11. Pouget E, Tonnar J, Lucas P, Lacroix-Desmazes P, Ganachaund F, Boutevin B (2010) Well-architectured poly(dimethylsiloxane)containing copolymers obtained by radical chemistry. Chem Rev 110(3):1233-1277

12. Kahsai WT, Pham UHT, Sankaran JS, Iqbal SM (2012) Selfassembled synthesis and characterization of microchannels in polymeric membranes. J Appl Phys 024701-024708:112

13. McDonald JC, Duffy DC, Anderson JR, Chiu DT, Wu H, Schneller OJA, Whitesides GM (2000) Fabrication of microfluidic systems in poly(dimethylsiloxane). Electrophoresis 21(1):27-40

14. McDonald JC, Whitesides GM (2002) Poly(dimethylsiloxane) as a material for fabricating microfluidic devices. Acc Chem Res 35(7):491-499

15. Berezhkova GV (1969) Nitevidnie kristally (The whiskers), Nauka, Moskva, p 158 
16. Clarson SJ, Fitzgerald JJ, Owen MJ, Smith SD (2000) Silicones and silicone-modified materials, Oxford University Press, Oxford, England. ISBN 0-8412-3613-5. doi:10.1021/bk-2000-0729

17. Myakov VN, Chuprov AD (1993) Rus Pat 2059671

18. Killean RCG, Tollin P, Watson DG, Young DW (1965) Twinning in p-aminobenzoic acid. Acta Crystallogr 19(3):482-483

19. Alleaume M, Salas-Cimingo G, Decap J (1966) Structure de l'acide para-aminobenzoique. Acad Sci C 262:416-417

20. Lai TF, Marsh RE (1967) The crystal structure of p-aminobenzoic acid. Acta Crystallogr 22(6):885-893
21. Anthimolam S, Natarajan S (2007) 4-Carboxyanilinium $(2 \mathrm{R}, 3 \mathrm{R})$-tartrate and redetermination of the $\alpha$-polymorph of 4-aminobenzoic acid. Acta Crystallogr 63(9):0514o517

22. Myakov VN, Lopatin MA, Kurskii YA (2002) Some reactions of dimeric silicon phthalocyanines under alkaline conditions. J Porphyrins Phthalocyanines 6(5):336-339

23. Treushnikov VM, Chesnokov SA (2008) Single-stage processes of polymer products photochemical synthesis with optical accuracy. J Photochem Photobiol 196:201-209 